\title{
FIRST IMAGES OF A SOLAR FLARE AT MILLIMETER WAVELENGTHS
}

\author{
Adriana V. R. Silva, ${ }^{1}$ Stephen M. White, ${ }^{2}$ Robert P. Lin, ${ }^{3}$ Imke de Pater, ${ }^{1}$ \\ K. Shibasaki, ${ }^{4}$ Hugh S. Hudson, ${ }^{5}$ and Mukul R. Kundu ${ }^{2}$ \\ Received 1995 October 27; accepted 1995 December 5
}

\begin{abstract}
We present the first high spatial resolution images of a solar flare at millimeter wavelengths. On 1994 August 17, a GOES soft X-ray class M1 flare was observed by the Berkeley-Illinois-Maryland Array at $86 \mathrm{GHz}$ by the Nobeyama $17 \mathrm{GHz}$ array and by the Yohkoh spacecraft. The flare displayed both a prominent impulsive phase in microwaves and a gradual phase that lasted over 30 minutes. The millimeter data were taken only during the gradual phase. The millimeter images show a source with a size of $\sim 8^{\prime \prime}$, a peak brightness temperature of $\sim 10^{6}$ $\mathrm{K}$, and maximum optical depth of 0.09 . At both X-ray and radio wavelengths, the emitting region appeared to be compact $\left(\$ 20^{\prime \prime}\right)$. In soft X-ray, the images are resolved into two sources: one located at a footpoint and the other at the top of the flaring loop. The millimeter emission is consistent with the predicted free-free flux from an isothermal temperature $(\sim 14 \mathrm{MK})$ loop-top source, a multitemperature footpoint source with a hot $(\sim 22 \mathrm{MK})$, and a cold $(\sim 12 \mathrm{MK})$ component. Most $(80 \%)$ of the millimeter flux density originates from the top of the magnetic loop, and the footpoint contribution is only $20 \%$.

Subject headings: Sun: flares
\end{abstract}

\section{INTRODUCTION}

The millimeter emission from flares can be either thermal bremsstrahlung or nonthermal gyrosynchrotron radiation (Kundu et al. 1990). The nonthermal millimeter emission is produced by electrons with energies of approximately $1 \mathrm{MeV}$ (White \& Kundu 1992), the same electrons that are responsible for the $\gamma$-ray continuum (Kawabata et al. 1982). Nonthermal emission is generally produced in the impulsive flash phase of flares. Thermal millimeter emission, however, is thought to originate from the postflare loops filled with hot plasma, which are bright in soft X-rays.

A major problem with single-dish observations of flares at millimeter wavelengths is the strong background radiation from the quiet Sun (10,000 sfu at $3 \mathrm{~mm})$. Before 1985, small single dishes were used at millimeter wavelengths and only very large flares were detected. The background level can be reduced by using a large telescope that has a beamwidth much smaller than the solar diameter. The first high sensitivity and high time resolution observations at millimeter wavelengths were obtained by Kaufmann et al. $(1984,1985)$ using the $14 \mathrm{~m}$ Itapetinga telescope in Brazil. With the addition of a multibeam receiver, Hermann et al. (1992) were able to use this telescope to measure source centroid positions as accurately as $5^{\prime \prime}$.

Interferometers offer a powerful advantage for solar observations: by correlating the signal of two antennas, structures larger than the fringe spacing will be resolved out (White \& Kundu 1992). Thus, the quiet-Sun background (scale size of the solar disk) measured by an interferometer will be much lower. Nakajima et al. (1985) used an interferometer for solar observing at Nobeyama, achieving 10 sfu sensitivity at $80 \mathrm{GHz}$. Kundu et al. (1990) report the first high sensitivity (0.02 sfu)

\footnotetext{
${ }^{1}$ Department of Astronomy, University of California, Berkeley, CA 94720. 20742.

2 Department of Astronomy, University of Maryland, College Park, MD

3 Department of Physics and Space Sciences Laboratory, University of California, Berkeley, CA 94720.

${ }^{4}$ National Radio Observatory, Nobeyama, Japan.

5 Institute for Astronomy, University of Hawaii, Honolulu, HI 96822.
}

interferometric observations at $86 \mathrm{GHz}$ using the BerkeleyIllinois-Maryland Array (BIMA) composed of three $6 \mathrm{~m}$ dishes at the time.

BIMA has now been upgraded to six antennas spread out on an inverted $\mathrm{T}$ configuration, allowing mapping of the millimeter emission. We present in this Letter the first millimeter images of a solar flare, obtained using four of those antennas. We analyze the 1994 August 17 flare and obtain its location, size, and physical parameters. Comparison of the millimeter radiation with the flare emission at other wavelengths provides information on the millimeter-wave emission mechanism.

\section{OBSERVATIONS AND RESULTS}

The M1.5 flare $\left(\right.$ GOES flux $\left.=1.5 \times 10^{-2} \operatorname{ergs~} \mathrm{cm}^{-2} \mathrm{~s}^{-1}\right)$ described here was observed on 1994 August 17 shortly after $0100 \mathrm{UT}$. The flare heliocentric position was at S15 W35, in Active Region 7765 . This flare was simultaneously detected by BIMA at $86 \mathrm{GHz}$, by the $17 \mathrm{GHz}$ radioheliograph at Nobeyama Radio Observatory (NRO), and by the soft (SXT) and hard (HXT) X-ray telescopes on board Yohkoh. The hard $\mathrm{X}$-rays from $\mathrm{HXT}$, the $17 \mathrm{GHz}$ and total power observations at 2.0, 3.75, and 9.4 GHz from Nobeyama are discussed elsewhere (Silva et al. 1995).

The time profiles at different wavelengths are shown in Figure 1. The top panel presents the soft X-ray emission from SXT (stars) and the hard X-rays from the two lower energy channels of HXT: 14-23 keV (LO channel) and 23-33 keV (M1 channel). There was hardly any emission above $33 \mathrm{keV}$. The hard X-ray emission shows a small impulsive peak at 0102:21 UT and two broad peaks at 0105 and 0109 UT. By 0113 UT, all the hard X-ray emission has ceased. The soft $\mathrm{X}$-rays from the flare started to rise at around $0102 \mathrm{UT}$, reached maximum at approximately 0110 UT, and lasted for about 15 minutes.

The radio emission at 17 and $86 \mathrm{GHz}$ are shown in the bottom panel of Figure 1. The $17 \mathrm{GHz}$ flux from NRO shows a strong impulsive peak at 0102:19 UT, which lasted about 15 s, reaching 36 sfu. Unfortunately, BIMA was on a calibra- 

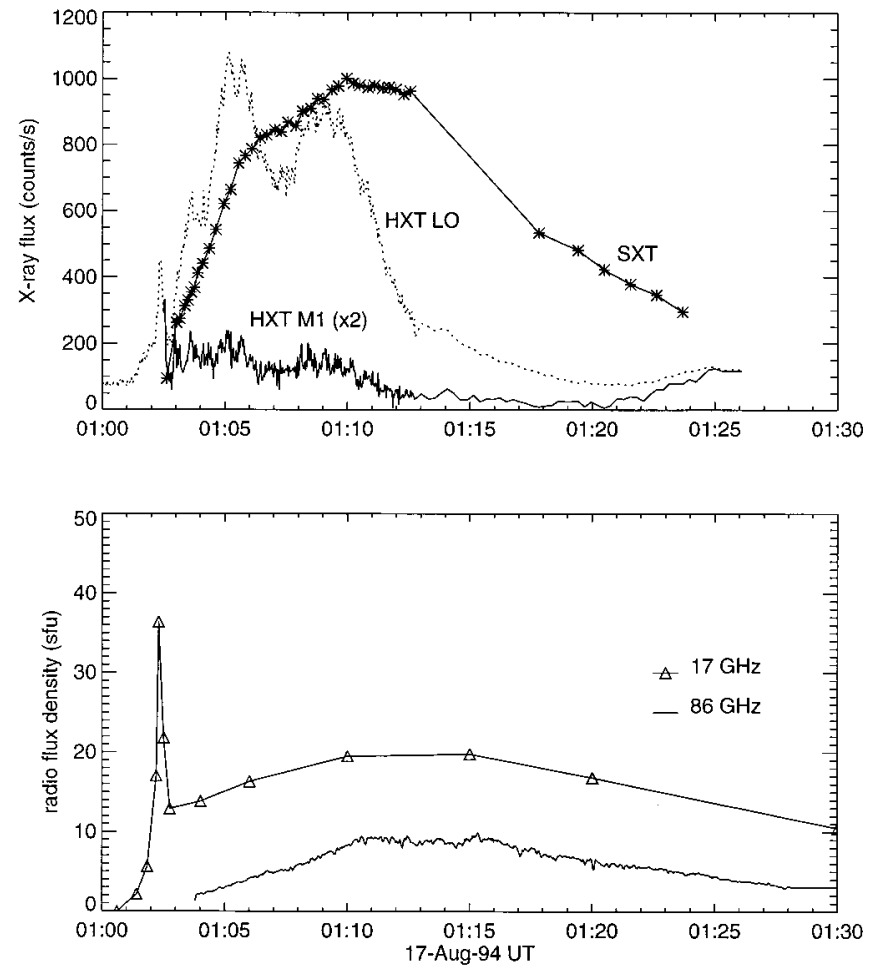

FIG. 1.-Time evolution of the different wavelength emissions of the 1994 August 17 flare. The soft and hard X-rays (LO and M1 channel) from SXT and HXT, respectively, are plotted on the top panel. The bottom panel shows the time profile of the NRO $(17 \mathrm{GHz})$ and BIMA $(86 \mathrm{GHz})$ radio emissions.

tion scan during this impulsive peak. Curiously, the impulsive peak, with such a strong signature at $17 \mathrm{GHz}$, produced only a very small peak in the HXT LO channel; it is about 2.5 times smaller than the maximum reached 3 minutes later. Nevertheless, this impulsive peak at 0102:21 UT is the strongest feature seen in the M1 channel count rates.

At $17 \mathrm{GHz}$, the gradual phase that followed reached a maximum of $20 \mathrm{sfu}$ around $0110-0115$ UT and lasted for 30 minutes. The $86 \mathrm{GHz}$ emission seems to follow the gradual 17 $\mathrm{GHz}$ emission, with a broad peak of 9 sfu at 0111-0115 UT, and lasts until 0140 UT. Note, however, that the absolute calibration for BIMA solar data is presently uncertain to within $50 \%$ due to the attenuation used to keep the strong solar signal from saturating the system.

Figure 2 (Plate L6) shows how the millimeter source relates spatially to the sources at other wavelengths at four different times (rows) throughout the flare. The columns correspond to maps of SXT, HXT LO, HXT M1, NRO (17 GHz), and BIMA $(86 \mathrm{GHz})$ from left to right, respectively. Preflare images are displayed on the top row (SXT and NRO only). The soft X-ray image at this time shows multiple loops, and the flare site (marked by the dashed cross hairs) is already bright. The same overall structure envelope is outlined in the preflare $17 \mathrm{GHz}$ map. During the flare, the same compact emitting region $\left(<20^{\prime \prime}\right)$ is seen in all the maps. The soft X-ray images show this region to be resolved into two sources of less than $10^{\prime \prime}$ each. The LO channel hard X-ray maps are $10 \mathrm{~s}$ integration, while the M1 maps needed $50 \mathrm{~s}$ integration because of its low count rates.

Since the flare occurred almost at sunset in California, the very elongated shape of the millimeter source seen in the
BIMA maps (last column of Fig. 2) is a consequence of the fairly elongated synthesized beam $\left(60^{\prime \prime} \times 6^{\prime \prime}\right)$. The intrinsic time resolution of the millimeter data is $2 \mathrm{~s}$. Since there was no temporal evolution observed in the millimeter maps, the images shown in Figure 2 were integrated over 2 minutes to increase their $(u, v)$ coverage. The calibration and mapping were done using the BIMA data reduction and analysis software package MIRIAD (Wright \& Sault 1993). The millimeter maps in Figure 2 show a single resolved source.

The millimeter data are modeled as a circular Gaussian source (the simplest extended source model). From this model, we determined the source size, total flux density, and brightness temperature as a function of time. The total flux density from the millimeter source as a function of time agrees well with the flux density measured at the shortest baseline (shown in Fig. 1), as it should. The source diameter (FWHM) is around $8^{\prime \prime}$. These sizes are consistent with the size of sources seen in the soft X-ray images.

The circular polarization of the $17 \mathrm{GHz}$ source measured by the Nobeyama array is very low $(<1 \%)$, indicating that the source was basically unpolarized. The millimeter observation has no polarization information.

\section{DISCUSSION}

The brightness temperature, $T_{b}$, of the source is determined from the total flux density, $S_{\nu}$, and the source size, $\Delta \Omega$ :

$$
S_{\nu}=\frac{2 k \nu^{2}}{c^{2}} \int T_{b} d \Omega
$$

The average $T_{b}$ is determined by using the total flux density and a source area of $\pi r^{2}$ ( $r$ is the radius of the source at half-power level). The average $T_{b}$ of the millimeter source reaches its peak value, $9 \times 10^{5} \mathrm{~K}$, at $0111 \mathrm{UT}$.

In order to determine if the $86 \mathrm{GHz}$ emission is thermal, we compare it with the free-free emission from the flare plasma that produces the soft X-ray observed by SXT. By dividing different SXT filter images, the temperature and emission measure, EM, are determined for every pixel in the SXT images (Vaiana, Krieger, \& Timothy 1973; McTiernan et al. 1993). The optically thin $(\tau \ll 1)$ free-free flux density is given by (White \& Kundu 1992)

$$
S_{\text {free }}=1.577 \times 10^{9} \frac{\mathrm{EM}_{55}}{\sqrt{T_{6}}}\left[17.29+\ln \left(T_{6}\right)-\ln \left(\nu_{\mathrm{GHz}}\right)\right] \mathrm{sfu},
$$

where $\mathrm{EM}_{55}$ is the emission measure in units of $10^{55} \mathrm{~cm}^{-3}, T_{6}$ is the temperature in millions of kelvins, and $\nu_{\mathrm{GHz}}$ is the frequency in gigahertz. The total radio flux density at $86 \mathrm{GHz}$ from the soft X-ray plasma calculated using eq. (2) is shown in Figure $3 a$ (triangles).

The gradual $17 \mathrm{GHz}$ emission after $0105 \mathrm{UT}$ is $\sim 30 \%$ higher than the total free-free flux density implied from the soft X-rays (Fig. 3a, triangles). The microwave spectra (2.0, $3.75,9.4$, and $17 \mathrm{GHz}$ ) from Nobeyama (discussed in detail in Silva et al. 1995) during the late stages of the flare are consistent with free-free emission from a much cooler $\left(10^{6} \mathrm{~K}\right)$ and extended ( $\sim 80^{\prime \prime}$ in diameter) source, since $S_{\text {free }} \propto V /\left(T_{e}\right)^{1 / 2}$ (where $V$ is the volume of the source). And indeed, emission from extended features can be seen in the $17 \mathrm{GHz}$ maps at later times (see Fig. 2).

The brightness temperature of the radio source is related to 

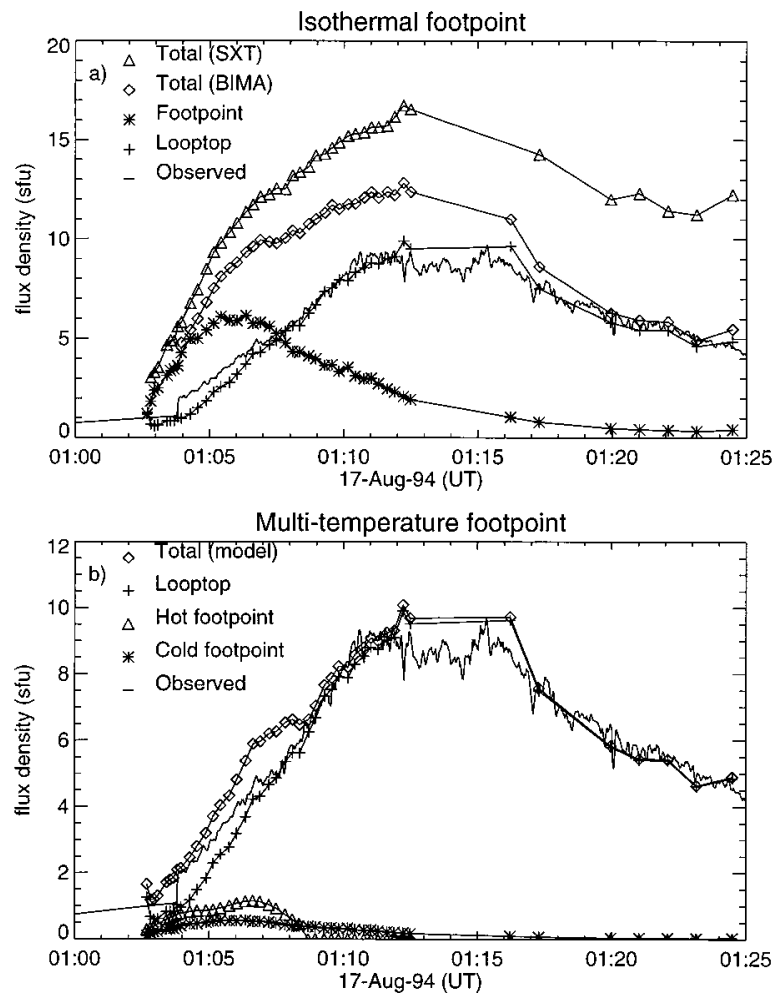

FIG. 3.-(a) Plot of the observed millimeter flux density from BIMA (solid line), the calculated free-free flux density from the soft X-ray plasma (triangles), and the BIMA output from the predicted free-free flux density (diamonds). The BIMA output is the flux density that BIMA should have seen from the hot plasma emitting the soft X-rays. Also plotted is the calculated radio flux density at $86 \mathrm{GHz}$ from the isothermal loop-top (crosses) and footpoint (stars) sources. (b) The same as above, only the footpoint source is not isothermal. The footpoint consists of a hot (triangles) and a cold (stars) components. The total predicted free-free flux from the footpoint and loop-top sources is shown as diamonds.

the physical temperature of the radiating electrons, $T_{e}$, by $T_{b}=T_{e}\left(1-e^{-\tau}\right)$. Thus, the optical depth, $\tau$, of the millimeter source can be estimated if $T_{e}$ is also known. The temperature of the electrons, $T_{e}$, used is that obtained from SXT filter ratios. The resulting optical depth has the same time profile as the millimeter flux density and is 0.09 at maximum. Thus, the millimeter emission is indeed optically thin throughout the flare, as expected.

Assuming a source depth comparable to the millimeter source's diameter of $8^{\prime \prime}$, and a temperature of $10^{7} \mathrm{~K}$ (inferred from SXT observations), the maximum optical depth of 0.09 implies a maximum ambient density of $4.5 \times 10^{11} \mathrm{~cm}^{-3}$. This value is consistent with the density of $4.2 \times 10^{11} \mathrm{~cm}^{-3}$ estimated from the maximum emission measure of the soft X-ray source, which also has a diameter of 8 ". We would like to point out that the magnetic loop where this flare occurred is very compact and most likely resides at low coronal heights, where densities are higher.

BIMA being an interferometer, however, will "resolve out" (i.e., not detect) flux from extended $\left(\gtrsim 20^{\prime \prime}\right)$ features. Maps of the radio emission predicted by equation (2) at $86 \mathrm{GHz}$ were made from SXT temperature and emission measure maps. The morphology of these maps is very similar to the SXT brightness maps (first column of Fig. 2). This is expected, since $S_{\text {free }} \propto$ EM. In order to determine BIMA's response to this radio source, we folded the SXT predicted radio maps through the BIMA instrument response. The result, the total flux density that should be seen by BIMA, is plotted on Figure $3 a$ as diamonds. As expected, the output flux density that BIMA should detect (diamonds) is less than the total radio flux density contained in the SXT radio maps (triangles), implying that BIMA would indeed resolve out some of the flux from the extended structures in the SXT radio maps. The observed flux density at $86 \mathrm{GHz}$ (Fig. 1, bottom) is plotted as a solid line on Figure $3 a$. Note that the agreement between these two fluxes (diamonds and solid line) is very good after 0117 UT. The resolved SXT predicted radio flux density (diamonds), however, is $\gtrsim 40 \%$ higher than the BIMA flux density during the rise of the millimeter flux density.

At close inspection, the soft X-ray maps show two sources not resolved by BIMA (not distinguishable in Fig. 2): an early compact source, which we have identified as the footpoint of a magnetic loop, and a more gradual source, which dominates the soft X-ray emission at later times (after 0108 UT). Since the gradual source overlies a neutral line seen in Kitt Peak magnetograms, it is thought to be at the top of the loop. Light curves of the sources show that the impulsive footpoint source peaks at 0105 UT and its time evolution follows that of the HXT LO channel peak, which has a maximum at this time, while the gradual loop-top source has a broad maximum from 0110 to 0113 UT. The temperature of the isothermal footpoint source is on average $10 \mathrm{MK}$, while its emission measure is $5.3 \times 10^{48} \mathrm{~cm}^{-3}$ at maximum (0105 UT). The emission measure of the loop-top source lies in the range $(10-140) \times 10^{47}$ $\mathrm{cm}^{-3}$, and its temperature in the range 7-15 MK.

The free-free radio flux density at $86 \mathrm{GHz}$ predicted for each of these two sources using equation (2) are plotted on Figure $3 a$ as stars and crosses, respectively. There is good agreement between the observed millimeter emission and the inferred free-free flux density from the soft X-ray-emitting plasma at the top of the loop. This agreement suggests the possibility that BIMA only detected the loop-top source. Why was the footpoint source not detected by BIMA? Based on the SXT observations, we expected a flux density of $6 \mathrm{sfu}$, well above the instrument sensitivity. One possible explanation is that the soft X-ray emission coming from the footpoint source is not thermal, thus invalidating the temperature and EM calculations for this source. Nonthermal gyrosynchrotron emission from this source would probably be well below BIMA's detection threshold, due to the very soft spectrum of the hard X-rays (no photons detected above $33 \mathrm{keV}$ ).

In order to check the hypothesis that the soft X-rays from the footpoint source are nonthermal, we have modeled the X-ray emission (SXT and HXT) as being generated by an instantaneous nonthermal electron population with a double power-law energy distribution. The instantaneous electrons with energies below a break energy, $E_{b}$, were assumed to have a power-law spectrum with a slope $\delta_{s}$ (producing the soft $\mathrm{X}$-rays), whereas the electrons with energy above $E_{b}$ had a power-law index $\delta_{h}$ (responsible for the hard X-ray emission). This model and its results are presented in detail in Silva et al. (1995).

The calculated photon flux spectrum from this power-law electron energy distribution (Brown 1971; Lin 1974) was then compared with that observed by SXT and HXT. Despite the good agreement between the modeled photon flux spectrum and the observed one, the amount of energy contained in such a large number of electrons at low energies $(1-2 \mathrm{keV})$ is very 
high. The total injected energy at the footpoint is 3 orders of magnitude greater than the instantaneous thermal energy. Such a large energy injection should heat far more plasma than is observed. This is the major criticism made to a nonthermal origin of soft X-rays in other flares (Hudson et al. 1994). Thus, the footpoint soft X-ray emission is unlikely to be nonthermal.

Last, we investigate the possibility of a multitemperature footpoint source. Note that the free-free flux calculated using equation (2) assumes an isothermal source. We propose that the footpoint source consists of two components: a hot and a cold source. The Fe XXV soft X-ray line observations by the Bragg crystal spectrometer (BCS) on board Yohkoh indicate the presence of a $\sim 20-23$ MK source. Since BCS has no spatial information, we assume this hot source to be located at the loop footpoint. Thus, the temperature $(\sim 22 \mathrm{MK})$ and emission measure $\left(5-20 \times 10^{47} \mathrm{~cm}^{-3}\right)$ of the footpoint hot component is obtained from the Fe XXV data. The soft X-ray flux of the hot component was then subtracted from the total flux measured in various SXT energy bands. This residual flux is assumed to be generated by a cooler component. The temperature $(\sim 12 \mathrm{MK})$ and emission measure $\left(2-8 \times 10^{47}\right.$ $\mathrm{cm}^{-3}$ ) that characterize this cooler plasma are assumed to have the same temporal evolution as the "old" isothermal footpoint source and are scaled such that its soft X-ray flux matches the residual flux described above. The sum of the flux of the two components agrees with SXT observed flux from the footpoint source within $5 \%-10 \%$.

The predicted free-free flux density at $86 \mathrm{GHz}$ for each component of the footpoint source is shown in Figure $3 b$. The stars and triangles represent the cold and hot footpoint components, respectively. The loop-top source is the same isothermal source as before and is plotted as crosses in Figure $3 b$. The total free-free flux from the multitemperature footpoint plus loop-top (crosses) sources is shown as diamonds.
Now the predicted flux from the footpoint source is less than $2 \mathrm{sfu}$, and the agreement between the predicted (diamonds) and the observed millimeter (solid line) flux densities is very good throughout the flare. Because soft X-rays are sensitive to high temperatures, most of the soft X-rays from the footpoint source originate from the hot component. However, since the free-free flux density is proportional to $T_{e}{ }^{-1 / 2}$, the contribution of the hot component to the radio flux density is small.

The occurrence of a multitemperature source in a flare seems plausible because of the very little heat conduction across a magnetic field line, which may result in different temperatures from one magnetic field line to another. Furthermore, along a single field line, there must be a whole range of temperatures: from the chromospheric temperatures to hot coronal flare plasma temperatures.

In summary, we believe the millimeter emission observed by BIMA for this flare to be thermal bremsstrahlung from the same flaring plasma detected in soft X-rays. Most (80\%) of the millimeter flux density is produced at the top of the loop. There is strong evidence that the footpoint source is not isothermal but made up of a hot $(\sim 22 \mathrm{MK})$ and a cold $(\sim 12$ MK) component, which contribute less than $20 \%$ of the total flux density at $86 \mathrm{GHz}$.

We are grateful to W. J. Welch, the Director of BIMA, responsible for the development of the BIMA instrument and its upgrade, for allowing a flexible observing schedule (dependent on solar activity). A. V. R. S. would like to thank the hospitality of R. Ulrich and the UCLA Astronomy Division, where this research was conducted. A. V. R. S. acknowledges support by NSF grant AST 93-20238. Solar radiophysics at the University of Maryland is supported by NSF grant ATM 93-16972, by NASA grant NAG W-1541, and NASA/CGRO grant NAG 5-1450. The use of BIMA for scientific research is supported by NSF grant AST 93-14847.

\section{REFERENCES}

Brown, J. C. 1971, Sol. Phys., 18, 489

Herrmann, R., Magun, A., Costa, J. E. R., Correia, E., \& Kaufmann, P. 1992, Sol. Phys., 142, 157

Hudson, H. S., Strong, K. T., Dennis, B. R., Zarro, D., Inda, M., Kosugi, T., \& Sakao, T. 1994, ApJ, 422, L25

Kaufmann, P., Correia, E., Costa, J. E. R., Dennis, B. R., Hurford, G. J., \& Brown, J. C. 1984, Sol. Phys., 91, 359

Kaufmann, P., Correia, E., Costa, J. E. R., Zodi Vaz, A. M., \& Dennis, B. R. 1985, Nature, 313, 380

Kawabatta, K., Ogawa, H., Takakura, T., Tsuneta, S., Ohki, K., Yoshimori, M., Okudaira, K., Hirashima, Y., \& Kondo, I. 1982, in HINOTORI Symp. on Solar Flares, Inst. Space and Astronaut. Sci., Tokyo, 168
Kundu, M. R., White, S. M., Gopalswamy, N., Bieging, J. H., \& Hurford, G. J. 1990, ApJ, 358, L69

Lin, R. P. 1974, Space Sci. Rev., 16, 189

McTiernan, J. M., Kane, S. R., Loran, J. M., Lemen, J. R., Acton, L. W., Hara, H., Tsuneta, S., \& Kosugi, T. 1993, ApJ, 416, L91

Nakajima, H., et al. 1985, PASJ, 36, 371

Silva, A. V. R., White, S. M., Lin, R. P., de Pater, I., Shibasaki, K., \& Nakajima, H. 1995, in preparation

Vaiana, G. S., Krieger, A. S., \& Timothy, A. F. 1973, Sol. Phys., 32, 81

White, S. M., \& Kundu, M. R. 1992, Sol. Phys., 141(2), 347

Wright, M. C. H., \& Sault, R. J. 1993, ApJ, 402, 546 
PLATE L6

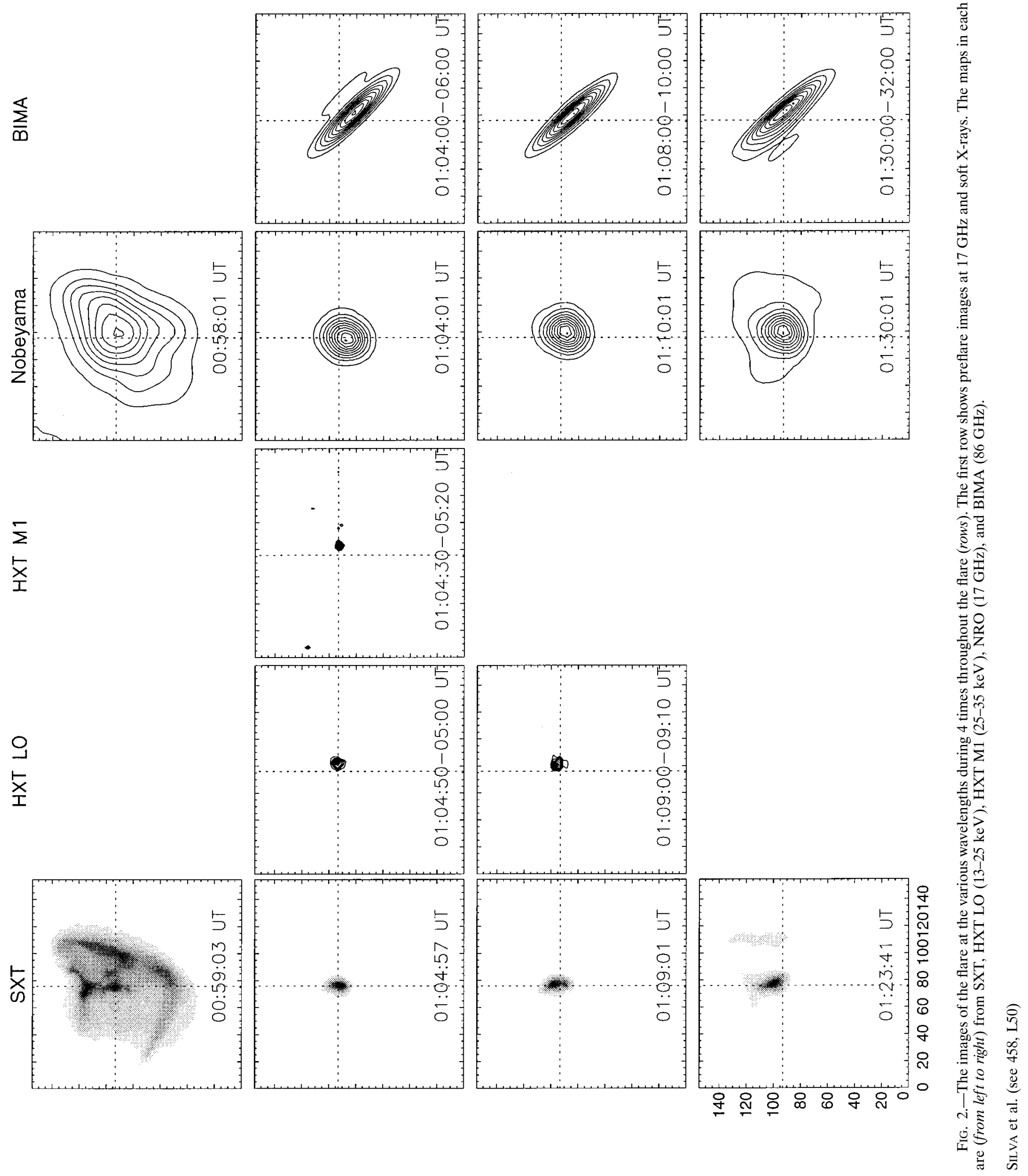

